\title{
Editorial
}

(BIRDEM Med J 2022; 12(1): 1-5)

\section{Non-communicable disease (NCDs)}

\section{Uddin KN}

Noncommunicable diseases (NCDs) now make up 7 of the world's top 10 causes of death, according to WHO's 2019 Global Health Estimates there is an increase from 4 of the 10 leading causes in 2000 . Heart disease remains the number 1 killer; diabetes and dementia enter the top 10. Strong primary health care is clearly the foundation on which everything rests, from combatting NCDs to managing a global pandemic."Member States voice historic support for a better and more sustainably funded WHO at 74th World Health Assembly, 31 ${ }^{\text {st }}$ May 2021.

$\mathrm{NCD}$ is a disease that is not transmissible directly from one person to another. NCDs include Parkinson's disease, autoimmune diseases, strokes, most heart diseases, most cancers, diabetes, chronic kidney disease, osteoarthritis, osteoporosis, Alzheimer's disease, cataracts, and others. NCDs, including heart disease, stroke, cancer, diabetes and chronic lung disease, are collectively responsible for almost $70 \%$ of all deaths worldwide. Almost three quarters of all NCD deaths, and $82 \%$ of the 16 million people who died prematurely, or before reaching 70 years of age, occur in low- and middle-income countries.About half were under age 70 and half were women. Every year, at least 5 million people die because of tobacco use and about 2.8 million die from being overweight. High cholesterol accounts for roughly 2.6 million deaths and 7.5 million die because of high blood pressure

\section{Risk factors}

They include age, gender, genetics, exposure to air pollution, and behaviors such as smoking, unhealthy diet and physical inactivity which can lead to hypertension and obesity, in turn leading to increased risk of many NCDs. Most NCDs are considered preventable because they are caused by modifiable risk factors. The WHO's World Health Report 2002 identified five important risk factors for non-communicable disease in the top ten leading risks to health. These are raised blood pressure, raised cholesterol, tobacco use, alcohol consumption, and being overweight. Diabetes has been included later in the list.It has been estimated that if the primary risk factors were eliminated, $80 \%$ of the cases of heart disease, stroke and type 2 diabetes and $40 \%$ of cancers could be prevented. In 2014, more than 1.9 billion adults were overweight; of these, over 600 million were obese. 42 million children under five years of age were overweight or obese in 2013.

\section{Environmental diseases}

The diseases of affluence are non-infectious diseases with environmental causes. Examples include: Many types of cardiovascular disease (CVD). Chronic obstructive pulmonary disease (COPD) caused by smoking tobacco. Diabetes mellitus type 2. Lower back pain caused by too little exercise.Malnutrition caused by too little food, or eating the wrong kinds of food (e.g. scurvy from lack of Vitamin C). Skin cancer caused by radiation from the sun.

\section{Inherited diseases}

Down syndrome is a change in chromosome numbers, Cystic fibrosis is defect in a gene caused by mutation.In an autosomal recessive disease which impairs the normal movement of sodium chloride in and out of cells, which causes the mucus-secreting organs to produce abnormally thick mucus. Cystic fibrosis affects the respiratory, digestive and reproductive systems, as well as the sweat glands.Huntington's, diseases a dominant genetic disease require the inheritance of one erroneous gene to be expressed.

Global health:Referred to as a "lifestyle" disease, because the majority of these diseases are preventable illnesses, the most common causes for noncommunicable diseases (NCD) include tobacco use (smoking), hazardous alcohol use, poor diets (high consumption of sugar, salt, saturated fats, and trans fatty acids) and physical inactivity..Historically, many NCDs were associated with economic development and 
were so-called a "diseases of the rich". The burden of non-communicable diseases in developing countries has increased however, with an estimated $80 \%$ of the four main types of NCDs - cardiovascular diseases, cancers, chronic respiratory diseases and diabetes now occurring in low- and middle-income countries. NCDs is attribute to 7 out of every 10 deaths in developing countries, killing 52 million people annually worldwide by 2030.Potential measures currently being discussed by the(World Health Organization)-Food and Agriculture Organization includes reducing the levels of salt in foods, limiting inappropriate marketing of unhealthy foods and non-alcoholic beverages to children, imposing controls on harmful alcohol use, raising taxes on tobacco, and legislating to curb smoking in public places.

\section{a. United Nations}

During the 64th session of the United Nations General Assembly in 2010, a resolution was passed to call for a high-level meeting of the General Assembly on the prevention and treatment NCDs with the participation of heads of state and government. The resolution also encouraged UN Member States to address the issue of non-communicable diseases at the 2010 Review Summit for the Millennium Development Goals.

\section{b. Global Non-communicable Disease Network:}

In 2009 the WHO announced the launch of the Global Non-communicable Disease Network (NCDnet). NCDnet will consist of leading health organizations and experts from around the world in order to fight against diseases such as cancer, cardiovascular disease, and diabetes WHO, said: "integrating the prevention of noncommunicable diseases and injuries into the national and global development agendas is not only achievable but also a priority for developing countries.

\section{c. NCD Alliance:}

The NCD Alliance is a global partnership founded in May 2009 by four international federations representing cardiovascular disease, diabetes, cancer, and chronic respiratory disease. The NCD Alliance brings together roughly 900 national member associations to fight noncommunicable disease. Long term aims of the Alliance include: 1. NCD/disease national plans for all 2. A tobacco free world 3. Improved lifestyles 4.Strengthened health systems 5. Global access to affordable and good quality medicines and technologies 6 . Human rights for people with NCDs.

\section{d. United Nations Interagency Task Force on the Prevention and Control of Non-communicable Diseases (UNIATF):}

The United Nations Interagency Task Force on the Prevention and Control of Non-communicable Diseases (UNIATF) was established by the United Nations Secretary-General in 2013 in order to provide scaled up action across the UN system to support governments, in particular in low- and middle-income countries, to tackle non-communicable diseases (NCDs).

\section{e. Young Professionals Chronic Disease Network:}

The Young Professionals Chronic Disease Network, or commonly referred to as YP-CDN, is a global network of roughly 5000 young professionals across 157 countries. The organization aims to mobilize these young people "to take action against social injustice driven by NCDs.".

The international community, the United Nations and the Organization of the American states have been analyzing the health and social impact of the Non Communicable Diseases in order to provide some concrete suggestions and intervention to reduce the burden to society, especially in low and medium income countries. On the UN side, the debate around the 2015 Sustainable Development Goals has raised interest in finding affordable solutions and policies. A few key points have been promoted as "best buy" interventions to be easily implemented in low and middle income countries, along with the recommendations from the World Health Organization (WHO).

\section{Risk factor \& intervention examples:}

1.Tobacco use: Tax increases, smoke free indoor workplaces and public places, health information and warnings, bans on tobacco advertising, promotion and sponsorship 2. Alcohol abuse:Tax increases, restricted access to retailed alcohol, bans on alcohol advertising 3. Unhealthy diet and physical inactivity: Reduced salt intake in food, replacement of trans fat with polyunsaturated fat, public awareness through mass media on diet and physical activity 4. Cardiovascular diseases (CVS) and Diabetes : Counseling and multidrug therapy for people with high risk of developing heart attacks and strokes (including those with establised CVD), treatment of heart attacks with aspirin 5.Cancer:Hepatitis B immunization to prevent liver cancer, screening and treatment of pre-cancerous lesions to prevent cervical cancer 
To support countries in their national efforts, WHO developed a Global action plan for the prevention and control of NCDs 2013-2020, which includes nine global targets that have the greatest impact on global NCD mortality. These targets address prevention and management of NCDs. ${ }^{1}$

Economics a.Previously, chronic NCDs were considered a problem limited mostly to high income countries, while infectious diseases seemed to affect low income countries. The burden of disease attributed to NCDs has been estimated at $85 \%$ in industrialized nations, $70 \%$ in middle income nations, and nearly $50 \%$ in countries with the lowest national incomes.. Given the global population distribution, almost $80 \%$ of deaths due to chronic NCDs worldwide now occur in low and middle income countries, while only $20 \%$ occur in higher income countries. China is expected to lose roughly \$558 billion in national income between 2005 and 2015 due to early deaths. In 2005, heart disease, stroke and diabetes caused an estimated loss of national income of 9 billion in India and 3 billion in Brazil. b.Absenteeism and presenteeism :The burden of chronic NCDs including mental health conditions is felt in workplaces around the world, notably due to elevated levels of absenteeism, or absence from work because of illness, and presenteeism, or productivity lost from staff coming to work and performing below normal standards due to poor health. For example, the United Kingdom experienced a loss of about 175 million days in 2006 to absence from illness among a working population of 37.7 million people. The estimated cost of absences due to illness was over 20 billion pounds in the same year. The cost due to presenteeism is likely even larger, although methods of analyzing the economic impacts of presenteeism are still being developed.

According to a study released by the World Economic Forum, the global cost of five non-communicable diseases will reach over $\$ 47$ trillion over the next twenty years - the diseases include CVD (cardiovascular disease), diabetes, mental illness, chronic respiratory disease, and cancer. The authors of the report wrote that $70 \%$ of lost output from non-communicable diseases are due to mental illness and cardiovascular diseases.

a.C ancer:For the vast majority of cancers, risk factors are environmental or lifestyle-related, thus cancers are mostly preventable NCD. Greater than $30 \%$ of cancer is preventable via avoiding risk factors including: tobacco, being overweight or obesity, low fruit and vegetable intake, physical inactivity, alcohol, sexually transmitted infections, and air pollution. Infectious agents are responsible for some cancers, for instance almost all cervical cancers are caused by human papillomavirus infection.

b. Cardiovascular diseases (CVDs) are the leading cause of death globally::An estimated 17.9 million people died from CVDs in 2019, representing 32\% of all global deaths. Of these deaths, $85 \%$ were due to heart attack and stroke.Over three quarters of CVD deaths take place in low- and middle-income countries.Out of the 17 million premature deaths (under the age of 70) due to noncommunicable diseases in 2019, 38\% were caused by CVDs.Most cardiovascular diseases can be prevented by addressing behavioural risk factors such as tobacco use, unhealthy diet and obesity, physical inactivity and harmful use of alcohol.

What are cardiovascular diseases:Cardiovascular diseases (CVDs) are a group of disorders of the heart and blood vessels. They include:coronary heart disease - a disease of the blood vessels supplying the heart muscle; cerebrovascular disease - a disease of the blood vessels supplying the brain;peripheral arterial disease - a disease of blood vessels supplying the arms and legs;rheumatic heart disease - damage to the heart muscle and heart valves from rheumatic fever, caused by streptococcal bacteria;congenital heart disease birth defects that affect the normal development and functioning of the heart caused by malformations of the heart structure from birth; and deep vein thrombosis and pulmonary embolism blood clots in the leg veins, which can dislodge and move to the heart and lungs.

In the early 2000 s, in which numerous studies have revealed a link between fast food and an increase in heart disease. These studies include those conducted by the Ryan Mackey Memorial Research Institute, Harvard University and the Sydney Center for Cardiovascular Health. Many major fast food chains, particularly McDonald's, have protested the methods used in these studies and have responded with healthier menu options. A fairly recent emphasis is on the link between low-grade inflammation that 
hallmarks atherosclerosis and its possible interventions. C-reactive protein (CRP) is a common inflammatory marker that has been found to be present in increased levels in patients at risk for cardiovascular disease, also osteoprotegerin which involved with regulation of a key inflammatory transcription factor called NF-êB has been found to be a risk factor of cardiovascular disease and mortality.

Hypertension six is a serious medical condition that significantly increases the risks of heart, brain, kidney and other diseases.An estimated 1.28 billion adults aged 30-79 years worldwide have hypertension, most (two-thirds) living in low- and middle-income countries. An estimated $46 \%$ of adults with hypertension are unaware that they have the condition.Less than half of adults (42\%) with hypertension are diagnosed and treated. Approximately 1 in 5 adults (21\%) with hypertension have it under control.Hypertension is a major cause of premature death worldwide.One of the global targets for noncommunicable diseases is to reduce the prevalence of hypertension by $33 \%$ between 2010 and 2030.

c. Diabetes:Type 2 Diabetes Mellitus is a chronic condition which is largely preventable and manageable but difficult to cure. Management concentrates on keeping blood sugar levels as close to normal ("euglycemia") as possible without presenting undue patient danger. This can usually be with close dietary management, exercise, and use of appropriate medications .Patient education, understanding, and participation is vital since the complications of diabetes are far less common and less severe in people who have well-managed blood sugar levels.

d. Chronic kidney disease:Although chronic kidney disease (CKD) is not currently identified as one of WHO's main targets for global NCD control, there is compelling evidence that CKD is not only common, harmful and treatable but also a major contributing factor to the incidence and outcomes of at least three of the diseases targeted by WHO (diabetes, hypertension and CVD

e. Chronic respiratory disease: According to the World Health Organization (WHO) hundreds of millions of people suffer daily from CRDs. Common CRDs are: Asthma, Chronic obstructive pulmonary disease, Occupational lung disease, and Pulmonary hypertension.. The main risk factors for developing CRDs are: tobacco smoking, indoor and outdoor air pollution, allergens, and occupational risks.

WHO helped launch the Global Alliance against Chronic Respiratory Diseases (GARD) in 2006. GARD is voluntarily composed of national and international organizations and works toward "reducing the global burden of chronic respiratory diseases" and focus mainly on vulnerable populations and low and middle-income countries.

In 2015 WHO published a list of countries ranking dath rate in non communicable disease.South Korea had the lowest rate (8.3\%), Japan (8.8\%), UK (11\%), USA(13.6\%), Colombia, Albania 14.6 and 14.7\%.

The WHO Global NCD Action Plan 2013-2020 follows on from commitments made by Heads of State and Government in the United Nations Political Declaration on the Prevention and Control of NCDs (resolution A/ RES/66/2), recognizing the primary role and responsibility of Governments in responding to the challenge of NCDs and the important role of international cooperation to support national efforts.

NCD In Bangladesh: NCDs account for an estimated $59 \%$ of total deaths in Bangladesh $-886,000$ deaths a year. In Bangladesh, $48 \%$ of men smoke; $20 \%$ of men and $32 \%$ of women have raised blood pressure. There were 7.1 million cases of diabetes in Bangladesh in 2015; a further 3.7 million cases may go undiagnosed.An estimated 129,000 deaths were attributed to diabetes in 2015. (WHO-NCD country profile 2014c, image) Bangladesh adopts multisectoral action plan for noncommunicable disease control and prevention. WHO supported Government of Bangladesh to adopt multisectoral action plan for prevention and control of noncommunicable diseases, a strategy spanning from 2018 to 2025 and involving nearly 30 ministries and agencies.

Noncommunicable diseases (NCDs) are one of the most urgent and complex public health challenges in Bangladesh. Cardiovascular diseases, diabetes, cancers and chronic respiratory diseases are responsible for $67 \%$ of all deaths in Bangladesh, many of which are premature. The national multisectoral action plan sets 
priorities for main stakeholders, being built in accordance with key programming documents such as the 7th Five Year Plan and the 4th Health, Nutrition and Population Strategic Investment Plan (HNPSIP) of the Government of Bangladesh. The action plan will be implemented in two phases, beginning with a focused three-year operational plan from 2018 to 2021. The multisectoral action plan is built on the evidence based approach that public policies of diverse sectors have an impact on health. In this regard, the health sector shall assume a central role in guiding, mobilizing and obtaining commitments from other sectors to develop policies which address shared risk factors for NCDs such as tobacco use, unhealthy diet, physical inactivity, harmful use of alcohol, and exposure to poor indoor air quality.Following the adoption of the plan, the first meeting of the National Multisectoral NCD Coordination Committee (NMNCC) was held in November 2018. Chaired by the Minister of Health and Family Welfare, several key decisions were taken in the meeting, including increasing human resources for the secretariat of the NMNCC, concluding memoranda of understanding with ministries of education and local government, and establishing local multisectoral coordination committees.For ensuring the sustainability and feasibility of the plan, the actions and activities are following the efficiency principle of implementing low cost measures with high health impact and are categorized into four major strategic areas: 1.advocacy, leadership and partnerships; 2.health promotion and risk reduction;3. health systems strengthening for early detection and management of NCDs and their risk factors; 4., monitoring, evaluation, and research. Govenment has opened NCD corners in each of 14000 community clinincs. The current action plan follows the successful implementation of past NCD control and prevention programmes and fulfills commitments that the Government of Bangladesh has made to the United Nations General Assembly at successive high level meetings on NCDs.Bangladesh has a total of 11 NCD programs at varying levels of development. Roughly half of the programs involved diabetes; three addressed the reduction of primary risk factors and about half provided infrastructure (e.g., hospitals or clinics) for NCD services or health professional training.Bangladesh, over the years had little success in effectively addressing the growing burden of noncommunicable diseases. Weak health system is hampering delivery of NCD care at PHC, and tracking of indicators is difficult due to lack of electronic database. Implementation of NCD management protocol, ensuring availability of drugs, maintaining electronic database, and monitoring program and indicators are the way forward to achieve universal health coverage for NCD. It is imperative that future research critically assess the effectiveness of national NCD policies by monitoring their implementation and level of population coverage ${ }^{2}$ WHO remains committed to supporting the Government of Bangladesh to implement this plan and the much needed solutions to secure the health, social and economic wellbeing of the present and future generations of Bangladesh.

\section{Khwaja Nazim Uddin}

Professor

Department of Internal Medicine

BIRDEM General Hospital, Dhaka, Bangladesh

Email: knuddin61@gmail.com

\section{REFERENCES}

1. GBD 2015 Risk Factors Collaborators. Global, regional, and national comparative risk assessment of 79 behavioural, environmental and occupational, and metabolic risks or clusters of risks, 1990-2015: a systematic analysis for the Global Burden of Disease Study 2015. Lancet, 2016; 388(10053):16591724

2. Biswas T, Pervin S, Tanim MIA, Niessen L, Islam A. et al. Bangladesh policy on prevention and control of non-communicable diseases: a policy analysis. BMC Public Health 17, 582 (2017). https://doi.org/ 10.1186/s12889-017-4494-2. 